\title{
Listeriolysin genes: complete sequence of ilo from Listeria ivanovii and of lso from Listeria seeligeri
}

\author{
Albert Haas ', Martina Dumbsky and Jürgen Kreft \\ Institut für Genetik und Mikrobiologie. Unicersität Wïrzburg. Würzburg (Germany)
}

(Received 11 December 1991)

Key words: Thiol-activated cytolysin; Listeriolysin O: Cysteine motif; ( L.. ir anor ii ): ( L. seeligeri)

The complete DNA sequences coding for the thiol-activated cytolysins from listeria irmotii, ivanolysin $O$ (ILO) and for secligerolysin $\mathrm{O}$ (LSO) from Listeria seeligeri have been determined. The deduced amino acid sequences revealed that: (i) the primary translation products comprise 528 (ILO) and 530) (LSO) amino acids, respectively, (ii) ILO contains two cysteines. L.SO has a substitution in the conserved cysteine motif.

The thiol-activated cytolysins are a family of poreforming toxins from diverse genera of Gram-positive bacteria [16], most of which are secreted into the extracellular medium. These toxins include the species-specific types of listeriolysin $O$ produced by the three Listeria species which are hemolytic on blood ágar: Listeria monocytogenes (pathogenic for man and animals), Listeria ivanocii (animal pathogen) and Listeria seeligeri (apathogenic). Their listeriolysins have previously been characterized biochemically $[6,10]$, and it has also been shown that they vary in cytolytic activity. The gene for listeriolysin $O(h / y)$ from $L$. monocytogenes has been cloned and sequenced $[4,13]$ and homologous DNA sequences have been detested in the chromosome of the two other hemolytic Listeria species [11]. It has been firmly established, that listeriolysin $O$ (LLO) is an essential virulence factor of $L$. monocytogenes (reviewed in Refs. 2 and 3), which enables this facultative intracellular parasite [2,5] to escape from the phagosome of the invaded mammalian cell, e.g., macrophages or other phagocytes. $L$. iranoi ii has alsu been shown to replicate intracellularly, whereas the avirulent $L$. seeligeri does not $[5,8]$.

The sequence data reported in this paper have been submitted to the EMBL/Genbank Data Libraries under the accession numbers X60461 (ilo) and X60462 (lso).

1 Present address: UCLA, Molecular Biology Institute, 405 Hilgard Ave., Los Angeles, CA 90024-1570, USA.

Correspondence: J. Kreft, (from 16 March 1992 on) Biozentrum der Universität Würzhurg. Lehrstuhl Mikrobiologie, Am Hubland, W. 8700 Würzburg, Germany.
We have established genomic DNA libraries of $L$. seeligeri (SICC3379) and L. itanorii (ATCC19119) by ligating chromosomai DNA fragments from partial Sau 3A digests ( $L$. it anovii) or size-fractionated Hindlll digests ( $L$. seeligeri) into the plasmid vector pTZ18R [12]. Transformed recombinant $E$. coli DH5- $\alpha$ clones were screened by colony hybridization for homology to a hly-specific gene probe $(651$ bp Hindlll fragment from pLM47, Ref. 11). One of the positive clones yielded plasmid pAHA9, which by DNA sequencing with the dideoxy chain termination method was shown to contain the complete determinant (/so) for listeriolysin $O$ from $L$. seeligeri, termed seeligerolysin $O$ (LSO) (Fig. 1). Among more than 15000 recombinants from the $L$. ii anotii library, three positive clones were identified which contained overlapping inserts of different sizes, but which were too small to span the entire listeriolysin gene. The DNA sequence from all three recombinants was determined. The largest insert (from pAHA10) included 1029 base pairs from the carboxy-terminal moiety of the gene (ilo) for listeriolysin $\mathbf{O}$ from $L$. itanotii, ivanolysin $O$ (ILO). The other two independent inserts contained shorter segments from the identical ilo sequence. For both pAHA9 and pAHA10 the listerial origin of the inserted DNA was confirmed by Southern hybridization. Several attempts to detect the complete ilo gene in the gene bank failed, but by the polymerase chain-reaction (PCR) we could isolate, clone into pTZ18R and sequence the complete determinant (Fig. 1). The PCRprimer for the $5^{\prime}$ region was deduced from a previously determined upstream sequence (Kreft and Weber, unpublished). in addition the complete lso gene was 
reisolated by the same method in order to confirm the sequence data from the library.

The DNA sequence homologies between ilo/hly, lso/hly and ilo/lso were $78 \%, 77 \%$ and $76 \%$, respectively. The deduced amino acid sequences (Fig. 1) showed that the primary translation product of $i l o$ is a 528 amino acid protein of $58541 \mathrm{Da}$, that of lso comprises 530 amino acids (59 181 Da). We have previously determined the Noterminal amino acid sequence of mature HEO [10]; therefore the signal peptide cleavage site for $1 \mathrm{LO}$ was placed after Ala-24. The N-termini of mature LSO and LLO are not known, but signal peptides are preferentially cleaved after an alanine, which is also found in both LSO and LLO at position 24.

The deduced amino acid sequences were compared (Fig. 2) to the deduced sequence for LLO from $L$. monocytogenes [4,13]. ILO, LSO and LLO are highly homologous: identity ILO/LLO 80\%, LSO/LLO 82\%, ILO/LSO $76 \%$; similarity $91 \%, 90 \%$ and $86 \%$, respectively, with rather heterogeneous $\mathrm{N}$-termini. ILO shows one deletion at position 25 and LSO the insertion of one serine at position 33 . The analysis of the predicted signal peptide of LSO revealed that it differs significantly from the corresponding ILO and LLO sequences: LSO lacks one positive charge at position 3 (Ile versus Lys) and has one hydrophobic amino acid less in the core region. Therefore, the LSO signal peptide might be less effective as an export-directing sequence.

The most interesting findings from the analysis of the deduced protein sequences were: (i) that LSO has a non-conservative amino acid substitution (Phe versus

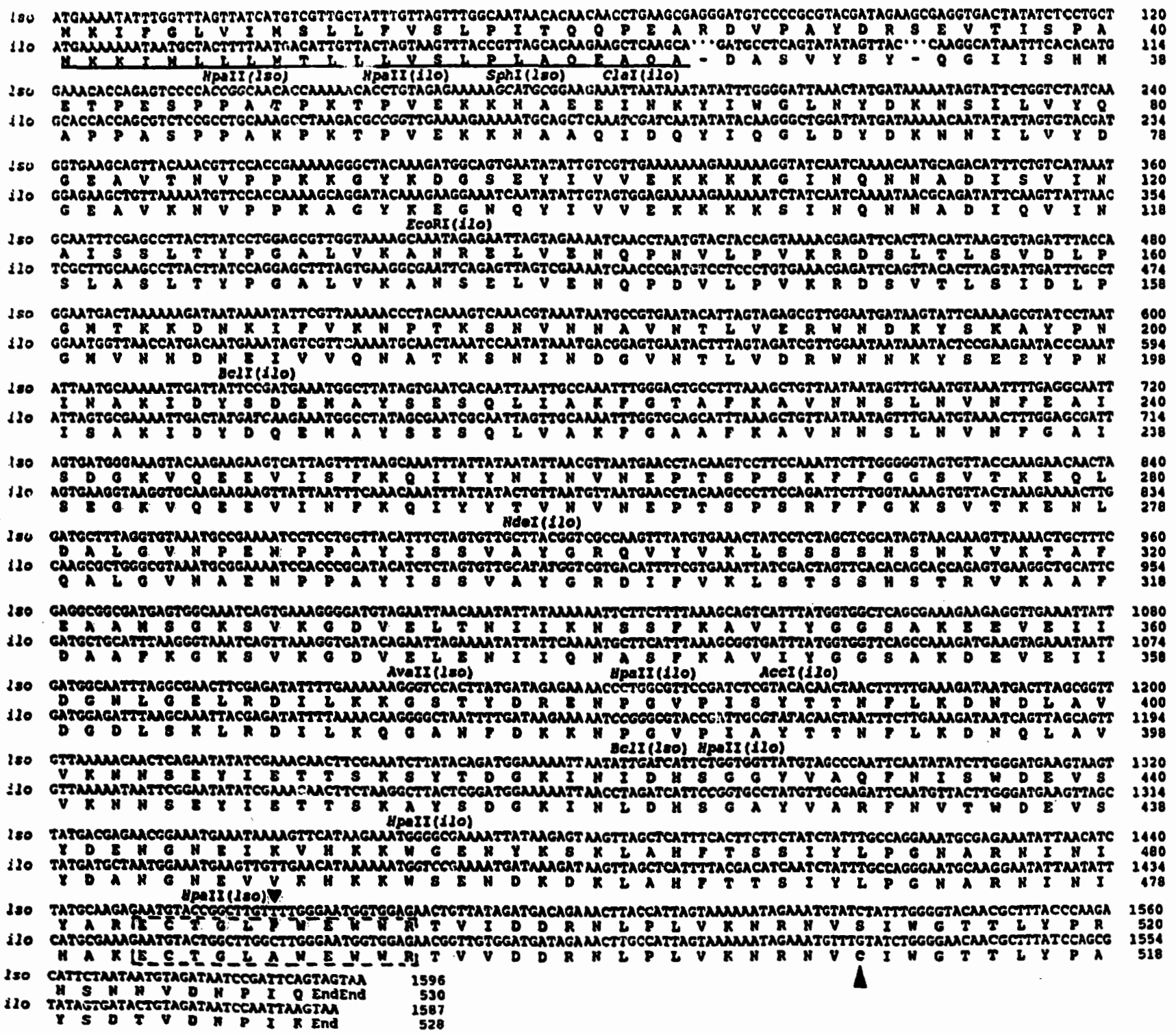

Fis. 1. Nucleotide and deduced amino acid sequences of $l s o(34.6 \% \mathrm{G}+\mathrm{C})$ and $i l o(35.5 \% \mathrm{G}+\mathrm{C})$. A few restriction sites, serving as landmarks, are indicated above the DNA sequence. The presumptive signal peptide is underlined, the conserved undecapetide is boxed with a broken line. Phe-489 in LSO and Cys-509 in ILO are indicated by arrows. 
Ala) at position 489 . Ala-489 is the sixth amino acid residue in the undecapeptide believed to be absolutely conserved among all thiol-activated cytolysins sequenced so far $[7,9,17,18]$, including $L L O$ from $L$. monocytogenes $[4,13]$. By site-directed mutagenesis it has been demonstrated by others that the integrity of this domain, and in particular the presence of certain tryptophane residues, is crucial for the hemolytic activity of these toxins [1]; this is also true for LLO from $L$. monocytogenes [14]. Although the normally conserved alanine is replaced in LSO by another hydrophobic, nonpolar, uncharged amino acid, the bulky aromatic ring of phenylalanine might have some detrimental effect on the hemolytic activity of LSO, compared to
LLO. This notion is supported by the fact that the phenylalanine is directly adjacent to the critical tryptophane residues. To ensure that the observed amino acid substitution in LSO did not result from a cloning artifact during the construction of pAHA9, the relevant chronosomal region of $L$. seeligeri was amplified by the polymerase chain reaction (PCR). The DNA sequence determined from the PCR product was identical to the one found for pAHA9. (ii) The conserved undecapeptide mentioned above is termed the 'cysteine motif', as it contains the single cysteine residue in all thiol-activated cytolysins analyzed so far. It has been reported that the presence of this cysteine is not absolutely required for the hemolytic activity of pneu-
Iro

LSO

LLO

IIO

Iso

LLO

IIO

ISO

LLO

IIO

LSO

IIO

ISO

IIO

LSO

LIO

IIO

ISO

LIO

IIO

Iso

LIO

IIO

LSD

LIO

ILO

LSO

LTO

IIO

ISO

ULO

IIO

ISO

LIO

IIO

LLO

مآ

LSO
LLO

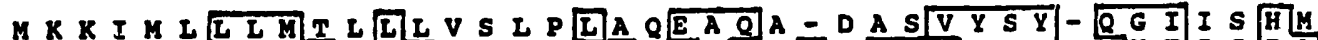

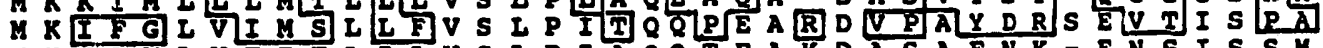
K KIVIV FITI I L S I P I A Q Q TEA K D A S A F N R - E N S I S S H

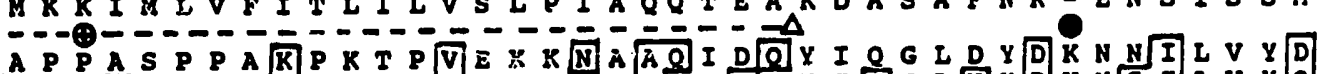
A A P P A S P P A S P K T P I E K K H A.DE I D K Y I Q G L D Y N K N N V L V Y H

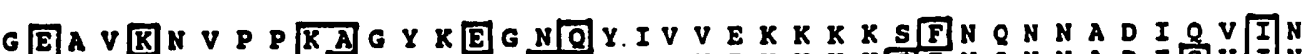

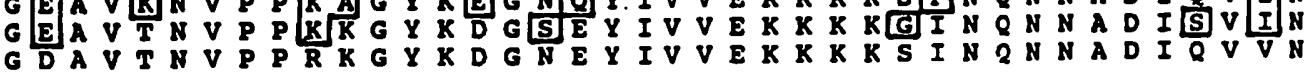

SIA S L T Y P G A L V KA N S E L V EN Q P D V L P V K R D S V T I S I D L P A IS S I T Y P G A I V K A N R E L V E N Q P D U I P V K R D S L T I S V D I P

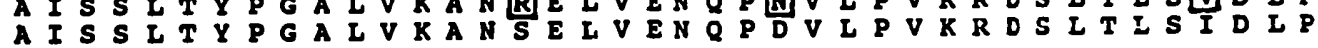

G M V]N国

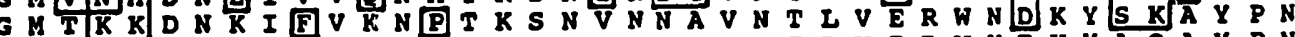
G T T T Q D N K I V V K N A T K S N V N N A V N T L V E R W N E K Y A Q A Y P N

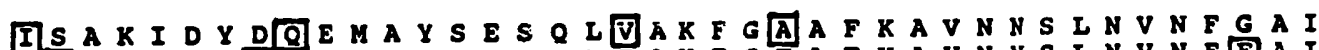
ILS A

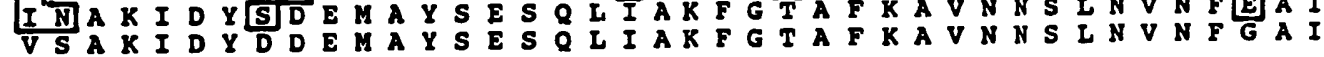

S E G K V Q E E V I D F K O I Y Y T V N V N E P T S P S R F F G K S V T K E 团 L $S$ E G K $Q$ E E V I S F K Q I Y Y N V N V N E P T R P S R F F G KA V T K E Q L

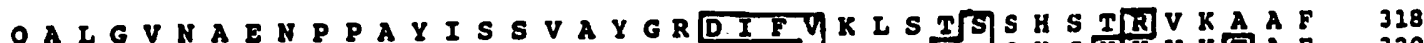
DA Q A I V N A E N P P A Y I S S V A Y G R Q Y Y I K I S T N S H S T Z̈ Y KA F

D A A FR] G K S V [KG D V E I E

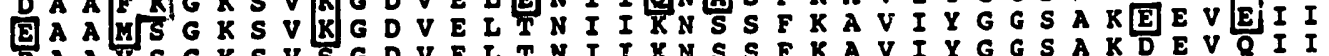

Fig. 2. Comparison of the deduced amino acid sequences for ILO and LSO with the LLO sequence (Refs. 4. 1.3). Regituss in ILO and/or LSO which are not identical to LLO are boxed. The presumptive signal peptide is indicated by a broken line. the position where LSO lacks one positive charge is marked. The additional Ser-33 in LSO is indicated by a black dot below the sequence. Phe-489 in LSO and Cys-5119 in ILO by arrows above. 
molysin [1], streptolysin O [15] and also listeriolysin $O$ (LLO) from $L$. monocytogenes [14]. Our results show that ILO contains two cysteine residues, one in the conserved region and another one 26 amino acids distal from there (position 509 in Figs. 1 and 2). Although all members of this group of toxins are oxygenlabile and thiol-activated only LO might be able to form intramolecular disulfide bonds upon oxidation. Further studies with the purified protein and with the isolated lso and ilo genes will show the significance of the differences described here to LLO and to the other toxins in this group.

We are grateful to $W$. Goebel for his continuing interest and support and to $\mathrm{K}$. Brehm for discussions and technical advice. $M$. Leimeister-Wächter and $T$. Chakraborty are thanked for kindly providing the $h l y$. specific gene probe, $M$. Wuenscher for his help in preparing the manuscript. This work was supported by grants from the Bundesministerium für Forschung und Technologie (BMFT $01 \mathrm{KI} \mathrm{88059)}$ and the Fonds der Chemischen Industrie (to J.K.). A.H. was recipient of a fellowship from the Boehringer Ingelheim Fonds.

\section{References}

I Boulnois. G.J., Mitchell, T.J., Saunders, F.K., Mendez, F.J. and Andrew, P.W. (1990) In Bacterial Protein Toxins (Rappouli, R. et al.. eds.). pp. 43-51, Fischer, Stuttgart.
2 Chakraborty, T. and Goebel, W. (1988) Curr. Top. Microbiol. Immunol. 138, 41-58.

3 Cossart, P. and Mengaud, J. (1989) Mol. Biol. Med. 6, 463-474.

4 Domann, E. and Chakraborty, T. (1989) Nucleic Acids Res. $17(15), 6406$.

5 Gaillard, J.L., Berche, P., Mounier, J., Richard, S. and Sansonetti, P. (1987) Infect. Immun. 55, 2822-2829.

6 Geoffroy, C., Gaillard, J.L., Alouf, J.E. and Berche, P. (1989) J. Gen. Microbiol. 135, 481-487.

7 Geoffroy, C., Mengaud, J., Alouf, J.E. and Cossart, P. (1990) J. Bacteriol. 172, 7301-7305.

8 Hof, H. and Hefner, P. (1988) Infection 16 (S2), 141-144.

9 Kehoe, M.A., Miller, L., Walker, J.A. and Boulnois, G.J. (1987) Infect. Immun. 55, 3228-3232.

10 Kreft, J., Funke, D., Haas, A., Lottspeich, F. and Goebel, W. (1989) FEMS Microbiol. Lett. 57, 197-202.

11 Leimeister-Wächter, M. and Chakraborty, T. (1989) Infect. Immun. 57, 2350-2357.

12 Mead, D.A., S7.czesna-Skorupa, E. and Kemper, B. (1986) Prot. Eng. 1. 67-74.

13 Mengaud, J., Vicente, M.F., Chenevert, J., Moniz Pereira, J., Geoffroy, C., Gicquel-Sanzey, B., Baquero, F., Perez-Diaz, J.C. and Cossart, P. (1988) Infect. Immun. 56, 766-772.

14 Michel, E., Reich, K.A., Favier, R., Berche, P. and Cossart, P. (1990) Mol. Microbiol, 4, 2167-2178.

15 Pinkney, M., Beachey, E. and Kehoe, M. (1989) Infect. Immun. 57. 2553-2558.

16 Smyth, C.J. and Duncan, J.L. (1978) In Bacterial Toxins and Cell Membranes (Jeljaszewicz, J. and Wadström. T.. eds.), pp. 129183, Academic Press, London.

17 Tweten, R.K. (1988) Infect. Immun. 56, 3235-3240.

18 Walker, J.A., Allen, R.L., Falmagne, P., Johnson, M.K. and Boulnois. G.J. (1987) Infect. Immun. 55, 1184-1189. 